\title{
Quelques spécificités du problème des incertitudes en géotechnique
}

JEAN-PIERRE MAGNAN

Laboratoire central des ponts et chaussées 58 , bd Lefebvre

75732 Paris Cedex 15
L'auteur introduit par des exemples les sources usuelles d'incertitudes de la géotechnique: manque de chance dans l'implantation des sondages, erreurs humaines dans la manipulation ou l'interprétation des données, remaniement des éprouvettes de laboratoire... Dans ce contexte, la caractérisation des incertitudes, définies comme l'écart entre le modèle de l'ingénieur et la réalité, est une entreprise complexe, qui ne se réduit pas à l'application de simples analyses statistiques ou probabilistes. Il en est de même pour la théorie de la décision, qui nécessite des schémas d'analyse plus complexes que ceux que l'on avait appliqués dans les années 1960-1970. Néanmoins, ces techniques d'analyse doivent être connues pour éclairer la réflexion des ingénieurs.

Mots-clés : sources d'incertitude, analyse de risque, fondations, erreur.
NDLE: Les discussions sur cet article sont acceptées jusqu'au $1^{\text {er août } 2001 .}$
The author describes, by means of examples, the usual sources of uncertainty in geotechnical engineering: bad luck when choosing the location of soundings, human errors during data handling or interpretation, remoulding of samples before laboratory testing... In such a context, characterising uncertainties, defined as the distance between the engineer's model and reality, cannot be restricted to the use of elementary statistical or probabilistic techniques of analysis. The situation is the same for the decision theory, which needs more complex models or analysis than those used in the 1960-1970'ies. Nevertheless, these techniques must be known to engineers, in order to help them thinking more efficiently.

Key words : sources of incertainly, risk analysis, foundations, errors. 


\section{Introduction}

Parmi les sciences de l'ingénieur, la géotechnique a une spécificité lourde de conséquences: l'ingénieur chargé de l'aménagement d'un site ou de la conception d'un ouvrage peut spécifier une partie des matériaux qu'il utilise, comme le béton, l'acier, le bois, les matières plastiques... Mais il doit s'accommoder des terrains existant sur le site et, pire encore, commencer par les identifier et les caractériser avant d'entreprendre la conception du projet.

L'identification des sols et des roches s'effectue au moyen de sondages (linéaires), d'essais ponctuels et de mesures géophysiques sur des surfaces ou des volumes. Comme le volume et le budget des reconnaissances sont toujours limités, la connaissance du site d'un projet reste partielle et les données des calculs sont incertaines.

L'intérêt des spécialistes de la géotechnique pour la description statistique des sols et des roches et pour les calculs de probabilités est né de cette incertitude, qui ne traduit pas un état aléatoire de la nature mais le niveau de connaissance ou de méconnaissance que l'on en a.

Le géotechnicien observe la nature à une échelle où il n'y a pas de doute sur la présence et les propriétés des matériaux. Dans chaque volume élémentaire, qui est infiniment grand par rapport aux atomes ou aux rayonnements, il y a un matériau (ou plusieurs matériaux) dont l'état et le comportement sont parfaitement définis et que l'on peut mesurer si on le désire. Mais on ne peut prélever et caractériser tout le sol d'un site. Entre des points, des lignes, des surfaces ou des volumes oủ l'on fait des mesures, il reste des espaces dont les propriétés doivent être estimées. Dans un volume de sol ou de roche, la nature et les propriétés des matériaux sont donc des fonctions aux valeurs estimées, assimilables aux champs aléatoires de la théorie des probabilités parce qu'en chaque point de nombreuses valeurs sont possibles, même si une seule (inconnue) existe réellement.

Dans ce texte, la probabilité ne décrira pas la nature aléatoire de la matière mais plutôt, comme l'a posé Cornell au début des années 1960, le degré de confiance de l'ingénieur pour le modèle qu'il a construit des massifs de sols et de roches existant sur le site de son étude ou du comportement de l'ouvrage qu'il étudie.

Cette définition implique la nature, qui existe, et un modèle, qui la représente. Tous les ingénieurs font des modèles et chaque modèle a un écart par rapport à la réalité. Les modèles de la géotechnique peuvent être très représentatifs ou très incertains suivant la complexité du site, que l'on ne connaît généralement pas a priori et qu'il faut identifier en même temps que l'on fait les mesures.

Les différentes classes d'erreurs, d'incertitudes et d'aléas que l'on peut rencontrer en géotechnique ainsi que leur prise en compte possible sont détaillés dans l'article de J.-I. Favre (2000). Nous allons les introduire ici à travers quelques exemples. Puis nous examinerons, dans le cas des fondations sur pieux, le niveau de complexité nécessaire des analyses d'incertitudes en géotechnique, qui s'avèrent nettement plus complexes que les premiers travaux des années 19601970.

\section{Les surprises d'une plaine côtière}

La reconnaissance géotechnique d'un terrain de 25 ha $(500 \mathrm{~m} \times 500 \mathrm{~m})$ avait été organisée de façon méthodique, avec des sondages au pénétromètre statique et des sondages carottés avec essais de laboratoire (Fig. 1,a). Rien en surface n'indiquait de variabilité particulière des terrains et l'ingénieur chargé de l'étude établit un modèle simple du site, constitué de couches horizontales dont les propriétés étaient la moyenne des résultats des essais de laboratoire. Leur variabilité était faible et cé modèle servit au calcul des tassements du sol sous un remblai général dé quelques mètres de hauteur. Ce modèle était bien calé sur les essais œdométriques. Pourtant, il s'avéra complètement faux: le site était traversé, selon l'axe d'implantation des sondages carottés, par une vallée fossile remplie de sols compressibles (Fig. 1.b). Les essais œedométriques représentaient uniquement la vallée. Le reste du site ne comportait que des argiles surconsolidées anciennes, dans lesquelles la vallée avait été creusée.

Cet exemple illustre à la fois un problème d'échantillonnage et un problème d'interprétation des données. L'échantillonnage (le choix de la position des essais) conditionne la représentativité des données. Dans l'exemple présenté, ces données ne reflétaient pas le site entier. Tout géotechnicien est à la merci d'un tel "manque de chance $»$. En revanche, l'interprétation des données était incorrecte aussi, par inexpérience de l'ingénieur responsable des calculs de tassements: les sondages de pénétration statique permettaient de délimiter clairement la vallée fossile et de guider l'interprétation du site.

Les informations disponibles permettaient donc de définir le site mais pas par une analyse simple des seuls paramètres de compressibilité œdométrique, qui étaient ceux nécessaires au calcul.

L'analyse simultanée d'informations d'origine et de nature diverses est très souvent nécessaire dans les études géotechniques et peut nécessiter des techniques d'analyse complexes, telles les méthodes d'analyse factorielle comme l'analyse en composantes principales qui est présentée dans l'article de Pouget et al. (2000).

\section{2}

\section{De l'importance du transport des carottes de sol}

Sur un autre projet, où l'on voulait construire un barrage à travers la vallée d'un fleuve, les couches superficielles des sols, jusqu'à une profondeur d'une dizaine de mètres, étaient constituées d'argiles molles sensibles. Ces argiles étaient assez résistantes pour porter les quelques mètres de remblai constituant le barrage dans le lit majeur du fleuve. Néanmoins, le projet établi sur la base d'essais de laboratoire réalisés à quelques milliers de kilomètres conclut à la nécessité d'un barrage en palplanches accrochées à une ligne de pieux: le laboratoire avait déclaré que les argiles qu'il avait reçues étaient à l'état liquide et ne pouvaient donc porter aucune charge. 


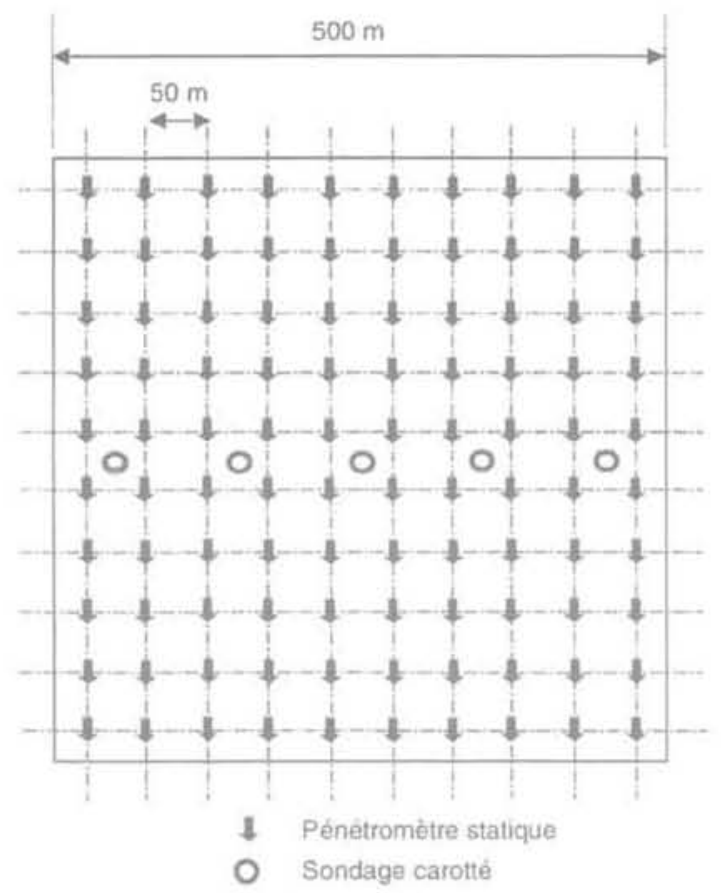

FG. 1a Implantation des sondages. Location of soundings.

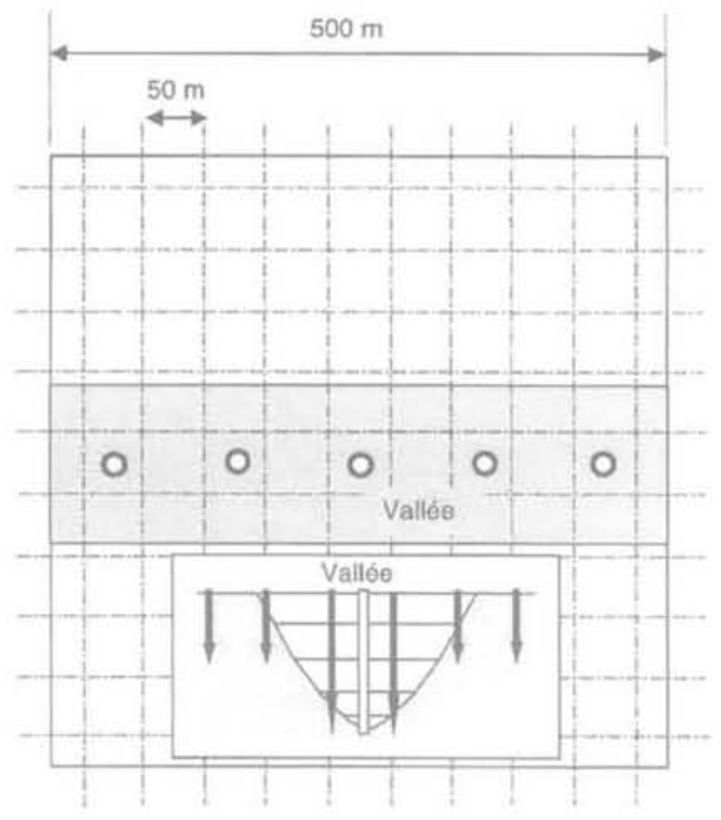

Vue de cóté de la vallée compressible

Fig.1 Plan schématique du site qui comportait une vallée fossile.

Schematic map of a site with a hidden valley.

Cet exemple montre l'importance de la qualité des mesures: si le résultat d'une mesure est faux (ici par remaniement préalable de l'éprouvette, mais cela peut venir de l'appareil de mesure ou d'une erreur de transmission des résultats), le modèle sera faux, même si tous les résultats des essais sont identiques.

\section{3}

\section{II ne faut pas toujours éliminer les données atypiques}

Une des démarches fréquentes des analyses statistiques consiste à éliminer les données qui perturbent l'analyse parce qu'elles sont trop différentes des autres. Dans certains cas, cela fait partie du modèle d'analyse: une pluie catastrophique ou un séisme de magnitude élevêe peuvent être traités comme des événements rares à longue période de retour et être éliminés de l'analyse des risques pour une période de durée limitée. Mais, quand il s'agit de propriétés des sols sur un site, il vaut mieux réfléchir soigneusement avant d'éliminer des données, comme l'illustre l'exemple suivant.

Sur le tracé d'une autoroute, la reconnaissance géotechnique avait révélé l'existence de deux couches de sols argileux compressibles, séparées par une couche de sable. La construction fut organisée en deux étapes, séparées de trois mois d'attente pour que la consolidation de l'argile ait le temps de s'effectuer. Lors de la mise en place du remblai de la deuxième phase, une rupture se produisit sur une centaine de mètres de longueur, retardant de plus d'un an l'achèvement des travaux. L'enquête réalisée après la rupture révéla que, dans la zone du glissement, la couche intermédiaire de sable était remplacée par une couche d'argile sableuse de perméabilité voisine de celle des couches d'argile. L'ingénieur responsable du projet avait bien noté que le sondage réalisé dans cette zone avait donné des résultats un peu différents des autres... Mais au lieu de demander des sondages de contrôle, il avait jugé que c'était une information aberrante et l'avait éliminée de son modèle du site.

Dans les reconnaissances géotechniques, il n'y a pas de raison que la nature soit systématiquement conforme à l'idée simplifiée que l'on se fait d'elle. La ressemblance d'une donnée aux autres n'est pas un critère de tri... Il faut vérifier si ce n'est pas plutòt un indice de la mauvaise qualité du modèle du site, que des données complémentaires pourront permettre d'améliorer.

\section{À propos d'erreurs grossières}

Lorsque l'on entreprend des analyses statistiques, on admet de facon plus ou moins consciente un certain nombre d'hypothèses, dont la première est qu'il existe des lois de probabilité des objets étudiés. L'image la plus fréquente de ces lois de probabilité est celle d'une fonction dont la valeur moyenne est connue en chaque point mais dont les valeurs réelles varient de façon aléatoire autour de cette valeur moyenne. La possibilité d'estimer les paramètres de la dérive (variation de la valeur moyenne) du paramètre étudié dans l'espace ou en fonction de la profondeur n'est pas une garantie de 
la validité du modèle. Ce n'est pas l'analyse statistique qui peut protéger l'ingénieur contre les fautes grossières, par nature non probabilisables, que l'on observe parfois dans les projets.

Un événement survenu lors de la reconnaissance géotechnique du tracé de la voie d'accès à une usine sidérurgique déjà construite a fait croire pendant plus d'un an que la construction de cette voie d'accès devrait être abandonnée: I'origine de cette crise était une simple erreur de numérotation des caisses contenant les carottes des sols prélevés sur le site: des échantillons d'argiles raides prélevés à plus de vingt mètres de profondeur furent échangés avec des échantillons d'argiles molles prélevés en surface. Les essais de compressibilité réalisés sur les carottes donnèrent des caractéristiques très faibles pour les sols prélevés en profondeur et des pressions de préconsolidation très inférieures à la contrainte effective initiale à cette profondeur. L'application mécanique des formules de calcul des tassements montrait que ces couches profondes auraient dû tasser de plusieurs mètres sous le poids des couches supérieures et sous leur propre poids. Il fallut plus d'un an pour que la vraisemblance des données géotechniques fût remise en cause, en s'appuyant sur des règles de cohérence géologique et géotechnique du site sans aucun rapport avec les données analysées.

Ce type d'erreurs, heureusement rare, n'est pas décelable par une simple manipulation des données. Les erreurs signalées dans les exemples précédents (mauvais choix des données à analyser, remaniement du sol avant les essais) sont également très rares et font partie de cette catégorie des erreurs humaines ou grossières que l'on ne sait pas traiter de façon statistique ou probabiliste. Les codes de bonne pratique et recommandations professionnelles tentent de les écarter par une exhortation à un travail de qualité, exécuté par du personnel formé et compétent, voire par la vérification du travail par une autre personne.

\section{5}

\section{L'incertitude sur les résultats des calculs dépend de facteurs complexes}

Les spécialistes de la géotechnique ne se contentent pas de créer des modèles de sites, ils calculent aussi des ouvrages de formes variées. On comprend facilement que si les données du calcul sont incertaines (aléatoires), le résultat du calcul le sera aussi. La théorie des probabilités dispose pour l'étudier de la notion de fonction aléatoire, qui n'est nullement contraire à la définition de la probabilité que nous avons adoptée: le résultat du calcul est aussi un modèle du comportement réel de l'ouvrage. L'ouvrage a un comportement unique (en général) et la probabilité est celle de l'égalité du calcul (incertain) avec ce comportement réel du sol et de l'ouvrage.

L'évaluation de l'incertitude sur le résultat d'un calcul est compliquée en pratique par l'existence de sources d'incertitudes autres que celles des données (Fig. 2) : en général, les modèles de calcul ne sont qu'une approximation de la réalité du fonctionnement mécanique des massifs de sols ou de roches et le modèle géométrique du massif peut être lui-même très approximatif. Les charges imposées à l'ouvrage par son environnement sont mal connues. Et l'ouvrage finalement obtenu dépend aussi des conditions de sa réalisation. On se trouve donc dans la situation d'estimer des incertitudes sur des résultats numériques de calculs qui ne décrivent que de façon approximative la réalité du site et des travaux.

De telles analyses nécessitent des outils complexes dont la validation est difficile et dont le développement rencontre pour cette raison de nombreux obstacles.
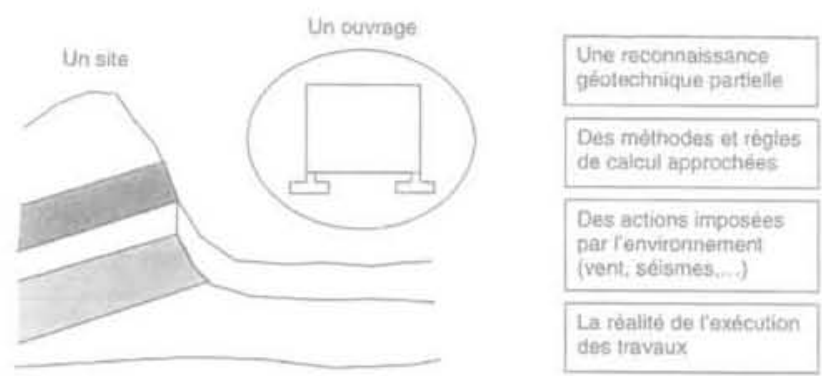

FIG:2 Les incertitudes des calculs géotechniques Uncertainly sources in geotechnical design.

\section{6}

\section{De l'utilité des corrélations}

La rareté des données géotechniques à l'échelle d'un projet courant oblige l'ingénieur à utiliser toute l'information existante, même si elle ne se rapporte qu'indirectement aux phénomènes qui conditionnent la conception et le fonctionnement de l'ouvrage. Les corrélations entre paramètres contribuent à la valorisation de cette information en permettant de contrôler la vraisemblance des résultats des essais, de détecter des anomalies, ou même de fabriquer les données de certains calculs.

L'utilisation de corrélations est facile... les techniques de recherche de corrélations par régression linéaire sont simples et les ouvrages spécialisés en proposent beaucoup. Il faut pourtant être très vigilant dans cette démarche et ne rechercher des corrélations qu'entre des paramètres dont la mécanique des sols ou des roches peut expliquer le lien.

\section{7}

\section{Évaluer les risques}

L'évaluation des risques est l'objectif affiché de beaucoup de recherches récentes dans lous les secteurs de la société. Le cadre d'analyse est la théorie de la décision, avec des schémas de causes et conséquences, des indices de fiabilité, etc. I.es outils sont fournis par les théories statistiques et probabilistes, parmi lesquelles on a observé au cours des dernières années l'émergence de la notion d'ensembles flous pour traiter les phénomènes difficiles à probabiliser. Les schémas d'analyse sont attendus des différentes disciplines concernées, c'est pourquoi il n'est pas éton- 
nant que les difficultés pratiques puissent différer d'un domaine à l'autre.

L'évolution des applications des probabilités et statistiques aux études de mécanique des sols, avec l'idée de définir des niveaux de risques associés aux ouvrages, s'avère assez différente des prévisions que l'on a pu faire au cours des années 1970. Le formalisme des méthodes d'analyse de risque et de décision, qui était facile à mettre en œuvre pour des calculs limités à quelques additions ou multiplications, n'a pas suivi l'évolution des méthodes de calcul modernes, mais on s'est aussi rendu compte que l'on manque d'éléments d'évaluation des niveaux de risques réels des ouvrages.

\section{8}

\section{L'ouvrage de référence}

Pour les ingénieurs et chercheurs qui se sont intéressés aux applications des statistiques et probabilités dans le domaine du génie civil et, plus particulièrement de la géotechnique, au cours des années 70 , un ouvrage récent servait de référence, le livre de Benjamin et Cornell (1970) sur les probabilités, les statistiques et la décision pour les ingénieurs civils (Fig. 3 ).

Dans leur préface, les auteurs écrivaient que: «Ce livre est destiné aux étudiants, praticiens, enseignants et chercheurs en génie civil... (Il s'appuie sur) l'utilisation d'exemples et de problèmes tirés du domaine du génie civil... La raison principale de ce livre (est qu') une nouvelle théorie, encore controversée, se développe autour d'un objectif de prise de décision économique....

De fait, ce livre avait pour axe central les outils de la

\section{Probability, Statistics, and Decision for Civil Engineers}

\section{Jack R. Benjamin $\quad$ C. Allin Cornell

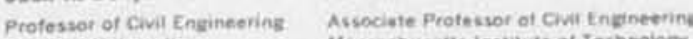 stantord University Massachuselts Institute of Technoiogs \\ Probabilités, statistiques et décision pour les ingénieurs civils \\ J.R. Benjamin, C.A. Cornell}

1. Analyse statistique des données

2. Éléments de la théorie des probabilités

3. Modèles probabilistes courants

4. Modèles probabilistes et données observées

5. Théorie élémentaire bayésienne de la décision

6. Analyse de décision des processus aléatoires indépendants

FiG.3 L'ouvrage de référence des années 70. The reference book of the 1970'sies. théorie de la décision. Sa lecture était et reste encore revigorante sur les objectifs qu'il est possible de poursuivre. Mais il est utile aussi de regarder les trente années passées et de se demander ce qui a évolué et pourquoi cette idée n'a pas débouché sur des applications pratiques. Nous l'examinerons sur le thème des fondations sur pieux.

A la page 527 de l'ouvrage, on trouve un exemple de problème servant de lien entre le livre de Benjamin et Cornell (1970) et le génie civil : un ingénieur doit sélectionner la longueur de pieux à enfoncer par battage jusqu'à un substratum dont la profondeur est incertaine. Les actions possibles sont de foncer des pieux de $20 \mathrm{~m}$ ou de $25 \mathrm{~m}$ et les profondeurs possibles du substratum sont de $20 \mathrm{~m}$ ou $25 \mathrm{~m}$ (Tableau I). Les conséquences des couples « décision-état» sont données dans le tableau 1. La solution explique comment prendre la meilleure décision. Une variante du problème consiste à inclure dans l'anaiyse les résultats d'un essai sonique de recherche du niveau du substratum, puis le battage préalable d'un pieu pour faire disparaitre l'incertitude.

TABLEAU Conséquences des actions suivant les états de la nature,

Consequences of actions depending on the states of nature.

\begin{tabular}{l|l|l}
\hline $\begin{array}{c}\text { Actions } \\
\text { États de la nature }\end{array}$ & \multicolumn{1}{|c|}{ Pieu de 20 $\mathrm{m}$} & Pieu de $25 \mathrm{~m}$ \\
\hline Rocher à $20 \mathrm{~m}$ & $\begin{array}{l}\text { Décision correcte, } \\
\text { pas de perte }\end{array}$ & $\begin{array}{l}5 \mathrm{~m} \text { de pieu } \\
\text { à couper } \\
\text { et enlever, perte } \\
\text { de } 100 \text { unités }\end{array}$ \\
\hline Rocher à $25 \mathrm{~m}$ & $\begin{array}{l}\text { Équipement } \\
\text { de battage } \\
\text { à modifier, } \\
\text { perte de } 400 \text { unités }\end{array}$ & $\begin{array}{l}\text { Décision } \\
\text { correcte, } \\
\text { pas de perte }\end{array}$ \\
\hline
\end{tabular}

L'ouvrage de Benjamin et Cornell définit clairement les fondements philosophiques de leur analyse. Pour eux. l'incertitude nait de la différence entre la réalité et le modèle qui la représente. Le modèle est l'idée que l'ingénieur se fait de la réalité, qu'elle provienne de l'analyse de mesures, de calculs ou d'idées "généralement admises ). On peut pour éclairer les idées citer comme exemples de modèles, le modèle géologique ou géotechnique d'un site, ou le résultat d'un calcul.

Le problème de cette approche est qu'elle ne s'intéresse pas au coeur de l'activité du géotechnicien, qui est la conception et la justification des projets d'ouvrages. Ainsi, dans l'exemple rappelé ci-dessus, le calcul du pieu se limite à "atteindre le substratum rocheuxn. C'est un type d'analyse géotechnique pour le moins très élémentaire. C'est probablement aussi le seul type d'analyse que l'on sache vraiment faire, trente ans plus tard.

\section{9}

\section{Les questions et compétences de l'an 2000}

Les trente années écoulées depuis la publication du livre de Benjamin et Cornell (1970) ont été l'occasion 
d'une prise de conscience que l'évaluation des incertitudes sur la conception et la justification des ouvrages était vraiment un problème complexe. On peut le voir sur le même exemple d'un pieu dont on doit justifier la capacité portante en suivant les règles de calcul en vigueur.

Selon les règlements ou normes en vigueur dans la plupart des pays, la justification d'un pieu (Fig. 4) soumis à l'action de charges externes $\vec{F}$ et porté par la résistance de pointe $\vec{Q}_{p}$ et le frottement latéral $\vec{Q}_{s}$

s'appuie sur deux conditions pour chaque combinaison de charge: une condition d'équilibre de la forme;

$$
\overrightarrow{\mathrm{F}}<\vec{Q}_{s}+\vec{Q}_{p} \text {, }
$$

et une condition sur les déplacements ou déformations du pieu:

Déplacement < seuil prescrit.

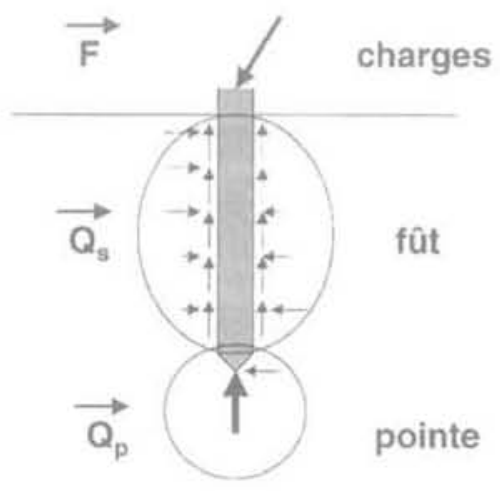

HG.4 Charges et résistance du sol sur un pieu. Loads and soil resistance on a pile.

Les procédures de calcul traitent globalement le problème et donnent les valeurs à comparer pour justifier l'ouvrage. Si l'on veut rechercher par contre les sources d'incertitudes, il faut identifier les modèles qui représentent les différentes réalités qui conditionnent le fonctionnement de l'ouvrage. Ces modèles correspondent aux charges, au comportement du sol, à l'interaction sol-pieu, à l'interaction pieu-structure, à la déformation du pieu, etc. On en vient alors à évaluer les écarts entre la réalité et chaque modèle, ce qui n'est pas facile à réaliser.

Les incertitudes sur les charges peuvent être rapportées aux combinaisons (arbitraires) des charges élémentaires et à ces charges élémentaires: masse des matériaux (géométrie et masse volumique), charges mobiles, reports de charges dus au fonctionnement mécanique de la structure, effets du vent, de la neige, des chocs, des séismes, du frottement négatif...

L'analyse des incertitudes sur les sols et sur les modèles de calcul est traitée différemment selon la méthode de calcul choisie. La méthode pressiométrique, d'usage très courant en France (Fig. 5), utilise des résultats de mesures mécaniques (l'essai pressiométrique) mais aussi des abaques qui expriment l'effet de la nature des sols et des types de pieux, et qui ont été calés sur l'expérience... La fiabilité d'une procédure de calcul dont certaines parties ont été calées sur l'expérience mais qui dépend tout de même d'essais ponctuels et de qualité inconnue pose des questions difficiles, d'autant que le calcul complet incorpore d'autres phénomènes (charges horizontales, effets de groupe, effet répartiteur de la rigidité de la structure portées par les pieux) qu'il faudrait aussi caractériser.

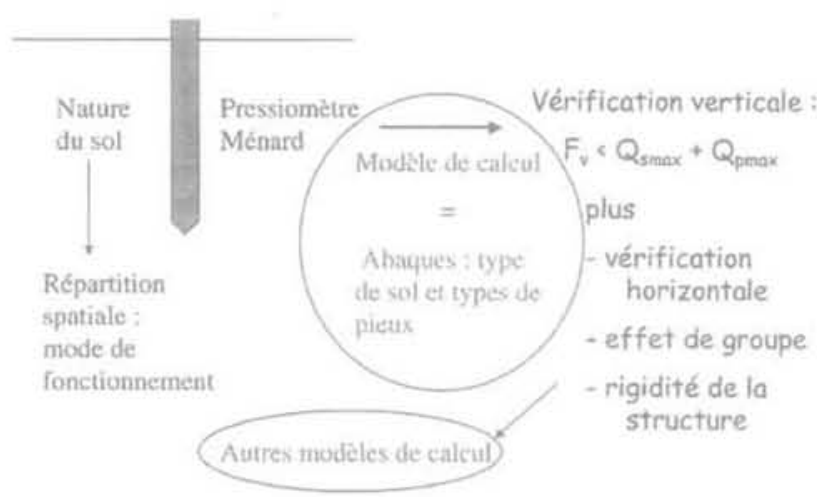

FIG. 5 Modèle d'interaction sol-pieu. Soil-pile interaction model.

L'utilisation d'autres modèles (méthodes de calcul utilisées au Pays-Bas, calcul en éléments finis...) oblige à refaire complètement l'analyse d'incertitudes.

Cet exemple à peine ébauché illustre à notre avis l'évolution des attentes des ingénieurs géotechniciens vis-à-vis des méthodes d'analyse statistique et probabiliste au cours des trente dernières années: les schémas de calcul des années 1970 étaient suffisamment simples pour permettre le déroulement d'une procédure d'analyse d'incertitude, voire de prise de décision... Mais ils sont trop simples par rapport aux développements modernes des connaissances sur le fonctionnement mécanique des pieux (et, de façon générale, de tous les ouvrages géotechniques). L'étalonnage global des méthodes de calcul, y compris les méthodes de mesure des paramètres et les méthodes d'exécution des travaux, n'a pratiquement été fait dans aucun pays, à part quelques exceptions comme l'étalonnage du calcul des pieux au pressiomètre et au pénétromètre statique en France. Cet étalonnage est indispensable pour évaluer le biais de la procédure de calcul (écart de la valeur moyenne calculée par rapport à la réalité). Sans cette vérification du bon cadrage de la procédure de calcul, il n'est pas possible d'évaluer les incertitudes et les risques associés aux ouvrages. Ce travail est long mais il devra être fait si l'on veut préciser les valeurs réelles des marges de sécurité en géotechnique et éventuellement les réduire un jour de façon volontaire et contrôlée. En l'absence d'un tel travail, l'application des procédures de dimensionnement validées par l'expérience reste le seul garant de la qualité du travail des ingénieurs.

\section{Conclusion}

Les études de géotechnique souffrent d'incertitudes dont les origines sont complexes. Nous avons cité l'interpolation ou l'extrapolation des données autour des points de mesure, la fiabilité des résultats de ces mesures, la complexité des problèmes étudiés et le volume réduit des informations disponibles, la caractérisation des résultats des calculs mais aussi l'existence 
d'incertitudes ou d'erreurs tellement importantes et inexplicables qu'elles perturbent les schémas classiques de l'analyse statistique ou probabiliste.

L'avenir des procédures d'analyse de risques et de prise de décision, connues sous le nom de théorie bayésienne de la décision, dépend de la possibilité d'inclure dans ces analyses les modèles modernes de fonctionnement des ouvrages. Nous possédons actuellement des règles historiques, partiellement justifiées et codifiées, dont l'utilisation garantit un résultat satisfaisant. Pourra-t-on affiner ces règles pour diminuer en moyenne la marge de sécurité? La réponse est incertaine : les outils d'analyse dépendent de données expérimentales complexes ou rares... La prévision est difficile dans un contexte de concurrence économique difficile à contrôler.

L'application des techniques d'analyse statistique et probabiliste présente donc un intérêt potentiel dans les différents domaines de la géotechnique et la connaissance de ces méthodes, mais aussi de leurs limites, est un élément important de la formation des ingénieurs de génie civil et de géotechnique.

\section{$\overline{\text { Bibliographie }}$}

Beniamin J.R. Cornell C.A. (1970) - Probability, statistics and decision for civil engineers. New York. Mc-Graw-Hill Book Company, $684 \mathrm{p}$.
Faure J.L. (2000) - $\alpha$ Les différents types d'erreur et leur prise en compte dans les calculs géotechniques i. Revue française de géotechnique, $n^{\circ} 93$, p. 11-20.
Pouget P. Cadoux N. Magnan J.P. (2000) $\propto$ Deux applications de l'analyse factorielle à des données qéotechniques i. Revué française de géotechnique, $\mathrm{n}^{\circ} 93$, p.43-54. 